\title{
THE WELL-POSEDNESS OF MIXED PROBLEM FOR ONE CLASS OF DEGENERATE MULTI-DIMENSIONAL HYPERBOLIC EQUATIONS
}

\author{
S. A. Aldashev \\ Institute of Mathematics and Mathematical Modelling, Ministry of Education and Science, \\ Kazakhstan, Almaty, E-mail: aldash51@mail.ru

\section{КОРРЕКТНОСТЬ СМЕШАННОЙ ЗАДАЧИ ДЛЯ ОДНОГО КЛАССА ВЫРОЖДАЮШИХСЯ МНОГОМЕРНЫХ ГИПЕРБОЛИЧЕСКИХ УРАВНЕНИЙ}

\author{
С. А. АЛДАШЕВ
}

Институт математики и математического моделирования МОН РК, Алматы, Казахстан, E-mail: aldash51@mail.ru

ABSTRACT. Oscillations of elastic membranes in 3D are modelled as degenerate multi-dimensional hyperbolic equations. For applied work, it is important to obtain explicit representations of solution of the studied boundary-value problems. This paper shows the unique solvability and obtains the explicit form of the classical solution of the mixed problem for degenerate multi-dimensional hyperbolic equations.

KEYWORDS: well-posedness, mixed problem, degenerate hyperbolic equations, Bessel function.

АннотАция. Колебания упругих мембран в трехмерном пространстве описывается вырождающимся многомерными гиперболическими уравнениями. При изучении приложений возникает необходимость получения явного представления решений исследуемых задач. В данной работе показана однозначная разрешимость и получен явный вид классического решения смешанной задачи для вырождающихся многомерных гиперболических уравнений.

КЛЮчЕВЫЕ СЛОВА: корректность, смешанная задача, вырождающиеся гиперболические уравнения, функция Бесселя.

\section{ВВЕДЕНИЕ}

Известно, что колебания упругих мембран в пространстве моделируется вырождающимся многомерными гиперболическими уравнениями $[1$, 2]. При изучении приложений возникает необходимость получения явного представления решений исследуемых задач. В работах автора $[3,4]$ доказаны существование и единственность классического решения задач Дирихле 
и Пуанкаре в цилиндрической области для вырождающихся многомерных гиперболических уравнений. Смешанная задача для этих уравнений в обобщенных пространствах изучена в $[5,6]$. Однако вопросы гладкости решений и его представления до сих пор не исследованы.

В данной работе для вырождающихся многомерных гиперболических уравнений с оператором Геллерстедта показана однозначная разрешимость и получен явный вид классического решения смешанной задачи.

\section{1. ПОСТАНОВКА ЗАДАЧИ И РЕЗУЛЬТАТ}

Пусть $D_{\alpha}$ - цилиндрическая область евклидова пространства $E_{m+1}$ точек $\left(x_{1}, \ldots, x_{m}, t\right)$, ограниченная цилиндром $\Gamma=\{(x, t):|x|=1\}$, плоскостями $t=\alpha>0$ и $t=0$, где $|x|$ - длина вектора $x=\left(x_{1}, \ldots, x_{m}\right)$.

Части этих поверхностей, образующих границу $\partial D_{\alpha}$ области $D_{\alpha}$, обозначим через $\Gamma_{\alpha}, S_{\alpha}, S_{0}$, соответственно.

В области $D_{\alpha}$ рассмотрим взаимно сопряженные вырождающихся многомерные гиперболические уравнения

$$
\begin{gathered}
L u \equiv t^{p} \Delta_{x} u-u_{t t}+\sum_{i=1}^{m} a_{i}(x, t) u_{x_{i}}+b(x, t) u_{t}+c(x, t) u=0, \\
L^{*} v \equiv t^{p} \Delta_{x} v-v_{t t}-\sum_{i=1}^{m} a_{i} v_{x_{i}}-b v_{t}+d v=0,
\end{gathered}
$$

где $p=$ const $>0, \Delta_{x}$ - оператор Лапласа по переменным $x=\left(x_{1}, \ldots, x_{m}\right)$, $m \geq 2$, a

$$
d(x, t)=c-\sum_{i=1}^{m} a_{i x_{i}}-b_{t} .
$$

В дальнейшем нам удобно перейти от декартовых координат $x_{1}, \ldots, x_{m}, t$ к сферическим $r, \theta_{1}, \ldots, \theta_{m-1}, t, r \geq 0,0 \leq \theta_{1}<2 \pi, 0 \leq \theta_{i} \leq \pi, i=2,3, \ldots, m-1$.

В качестве смешанной задачи рассмотрим задачу

Задача 1. Найти решение уравнения $(1)$ в области $D_{\alpha}$ из класса $C^{1}\left(\bar{D}_{\alpha}\right) \cap$ $C^{2}\left(D_{\alpha}\right)$, удовлетворяющее краевым условиям

$$
\left.u\right|_{S_{0}}=\tau(r, \theta),\left.\quad u_{t}\right|_{S_{0}}=\nu(r, \theta),\left.\quad u\right|_{\Gamma_{\alpha}}=\psi(t, \theta) .
$$

Пусть $\left\{Y_{n, m}^{k}(\theta)\right\}$ - система линейно независимых сферических функций порядка $n, 1 \leq k \leq k_{n},(m-2) ! n ! k_{n}=(n+m-3) !(2 n+m-2), \theta=$ $\left(\theta_{1}, \ldots, \theta_{m-1}\right), W_{2}^{l}\left(S_{0}\right), l=0,1, \ldots-$ пространства Соболева.

Имеет место ([7])

Лемма 1. Пусть $f(r, \theta) \in W_{2}^{l}\left(S_{0}\right)$. Если $l \geq m-1$, то ряд

$$
f(r, \theta)=\sum_{n=0}^{\infty} \sum_{k=1}^{k_{n}} f_{n}^{k}(r) Y_{n, m}^{k}(\theta),
$$

а такэе ряды, полученные из него дифференцированием порядка $p \leq l-$ $m+1$, сходятся абсолютно и равномерно. 
Лемма 2. Для того, чтобъ $f(r, \theta) \in W_{2}^{l}\left(S_{0}\right)$, необходимо и достаточно, чтобы коэффициенты ряда (3) удовлетворяли неравенствам

$$
\left|f_{0}^{1}(r)\right| \leq c_{1}, \sum_{n=1}^{\infty} \sum_{k=1}^{k_{n}} n^{2 l}\left|f_{n}^{k}(r)\right|^{2} \leq c_{2}, \quad c_{1}, \quad c_{2}=\text { const } .
$$

Через $\tilde{a}_{i n}^{k}(r, t), a_{i n}^{k}(r, t), \tilde{b}_{n}^{k}(r, t), \tilde{c}_{n}^{k}(r, t), \tilde{d}_{n}^{k}(r, t), \rho_{n}^{k}, \bar{\tau}_{n}^{k}(r), \bar{\nu}_{n}^{k}(r)$, и $\psi_{n}^{k}(t)$ обозначим коэффициенты разложения ряда (3), соответственно функций $a_{i}(r, \theta, t) \rho(\theta), a_{i} \frac{x_{i}}{r} \rho, b(r, \theta, t) \rho, c(r, \theta, t) \rho, d(r, \theta, t) \rho, \rho(\theta), i=1, \ldots, m, \tau(r, \theta)$, $\nu(r, \theta), \psi(t, \theta)$, причем $\rho(\theta) \in C^{\infty}(H), H$ - единичная сфера в $E_{m}$.

Пусть $a_{i}(r, \theta, t), b(r, \theta, t), c(r, \theta, t) \in W_{2}^{l}\left(D_{\alpha}\right) \subset C\left(\bar{D}_{\alpha}\right), l \geq m+1, i=$ $1, \ldots, m$.

Тогда справедлива

Теорема 1. Если $\tau(r, \theta), \nu(r, \theta) \in W_{2}^{l}\left(S_{0}\right), \psi(t, \theta) \in W_{2}^{l}\left(\Gamma_{\alpha}\right), l>\frac{3 m}{2} u$ выполняется условие

$$
\cos \mu_{s, n} \alpha^{\prime} \neq 0, s=1,2, \ldots
$$

то задача 1 имеет единственное решение, где $\mu_{s, n}$ - положительные нули функиий Бесселя первого рода $J_{n+\frac{(m-2)}{2}}(z), \alpha^{\prime}=\frac{2}{2+p} \alpha^{\frac{(2+p)}{2}}, n=0,1, \ldots$.

Далее докажем теорему 1.

\section{2. РАЗРЕШИМОСТЬ ЗАДАЧИ 1}

В сферических координатах уравнение (1) имеет вид

$$
\begin{gathered}
L u \equiv t^{p}\left(u_{r r}+\frac{m-1}{r} u_{r}-\frac{\sigma u}{r^{2}}\right)-u_{t t}+ \\
+\sum_{i=1}^{m} a_{i}(r, \theta, t) u_{x_{i}}+b(r, \theta, t) u_{t}+c(r, \theta, t) u=0,
\end{gathered}
$$

где

$$
\begin{gathered}
\sigma \equiv-\sum_{j=1}^{m-1} \frac{1}{g_{j} \sin ^{m-j-1} \theta_{j}} \frac{\partial}{\partial \theta_{j}}\left(\sin ^{m-j-1} \theta_{j} \frac{\partial}{\partial \theta_{j}}\right), \\
g_{1}=1, g_{j}=\left(\sin \theta_{1} \ldots \sin \theta_{j-1}\right)^{2}, j>1 .
\end{gathered}
$$

Известно [7], что спектр оператора $\sigma$ состоит из собственных чисел $\lambda_{n}=$ $n(n+m-2), n=0,1, \ldots$ каждому из которых соответствует $k_{n}$ ортонормированных собственных функций $Y_{n, m}^{k}(\theta)$.

Искомое решение задачи 1 будем искать в виде

$$
u(r, \theta, t)=\sum_{n=0}^{\infty} \sum_{k=1}^{k_{n}} \bar{u}_{n}^{k}(r, t) Y_{n, m}^{k}(\theta),
$$

где $\bar{u}_{n}^{k}(r, t)$ - функции, подлежащие определению. 
Подставив (6) в (5), умножив затем полученное выражение на $\rho(\theta) \neq 0$, и проинтегрировав по единичной сфере $H$, для $\bar{u}_{n}^{k}$ получим [8-10]

$$
\begin{gathered}
t^{p} \rho_{0}^{1} \bar{u}_{0 r r}^{1}-\rho_{0}^{1} \bar{u}_{0 t t}^{1}+\left(\frac{m-1}{r} t^{p} \rho_{0}^{1}+\sum_{i=1}^{m} a_{i 0}^{1}\right) \bar{u}_{0 r}^{1}+\widetilde{b}_{0}^{1} \bar{u}_{0 t}^{1}+\widetilde{c}_{0}^{1} \bar{u}_{0}^{1}+ \\
+\sum_{n=1}^{\infty} \sum_{k=1}^{k_{n}}\left\{t^{p} \rho_{n}^{k} \bar{u}_{n r r}^{k}-\rho_{n}^{k} \bar{u}_{n t t}^{k}+\left(\frac{m-1}{r} t^{p} \rho_{n}^{k}+\sum_{i=1}^{m} a_{i n}^{k}\right) \bar{u}_{n r}^{k}+\widetilde{b}_{n}^{k} \bar{u}_{n t}^{k}+\right. \\
\left.+\left[\widetilde{c}_{n}^{k}-\lambda_{n} \frac{\rho_{n}^{k}}{r^{2}} t^{p}+\sum_{i=1}^{m}\left(\widetilde{a}_{i n-1}^{k}-n a_{i n}^{k}\right)\right] \bar{u}_{n}^{k}\right\}=0 .
\end{gathered}
$$

Теперь рассмотрим бесконечную систему дифференциальных уравнений

$$
\begin{gathered}
\rho_{0}^{1} t^{p} \bar{u}_{0 r r}^{1}-\rho_{0}^{1} \bar{u}_{0 t t}^{1}+\frac{m-1}{r} t^{p} \rho_{0}^{1} \bar{u}_{0 r}^{1}=0, \\
\rho_{1}^{k} t^{p} \bar{u}_{1 r r}^{k}-\rho_{1}^{k} \bar{u}_{1 t t}^{k}+\frac{m-1}{r} t^{p} \rho_{1}^{k} \bar{u}_{1 r}^{k}-\frac{\lambda_{1}}{r^{2}} t^{p} \rho_{1}^{k} \bar{u}_{1}^{k}= \\
=-\frac{1}{k_{1}}\left(\sum_{i=1}^{m} a_{i 0}^{1} \bar{u}_{0 r}^{1}+\widetilde{b}_{0}^{1} \bar{u}_{0 t}^{1}+\widetilde{c}_{0}^{1} \bar{u}_{0}^{1}\right), \quad n=1, \quad k=\overline{1, k_{1}}, \\
\rho_{n}^{k} t^{p} \bar{u}_{n r r}^{k}-\rho_{n}^{k} \bar{u}_{n t t}^{k}+\frac{m-1}{r} t^{p} \rho_{n}^{k} \bar{u}_{n r}^{k}-\frac{\lambda_{n}}{r^{2}} t^{p} \rho_{n}^{k} \bar{u}_{n}^{k}= \\
=-\frac{1}{k_{n}} \sum_{k=1}^{k_{n-1}}\left\{\sum_{i=1}^{m} a_{i n-1}^{k} \bar{u}_{n-1 r}^{k}+\widetilde{b}_{n-1}^{k} \bar{u}_{n-1 t}^{k}+\left[\widetilde{c}_{n-1}^{k}+\right.\right. \\
\left.\left.+\sum_{i=1}^{m}\left(\widetilde{a}_{i n-2}^{k}-(n-1) a_{i n-1}^{k}\right)\right] \bar{u}_{n-1}^{k}\right\}, \quad k=\overline{1, k_{n}}, \quad n=2,3, \ldots
\end{gathered}
$$

Суммируя уравнение (9) от 1 до $k_{1}$, а уравнение (10) - от 1 до $k_{n}$, а затем сложив полученные выражения вместе с (8), приходим к уравнению (7).

Отсюда следует, что если $\left\{\bar{u}_{n}^{k}\right\}, k=\overline{1, k_{n}}, n=0,1, \ldots$ - решение системы (8)-(10), то оно является решением уравнения (7).

Нетрудно заметить, что каждое уравнение системы (8)-(10) можно представить в виде

$$
t^{p}\left(\bar{u}_{n r r}^{k}+\frac{m-1}{r} \bar{u}_{n r}^{k}-\frac{\lambda_{n}}{r^{2}} \bar{u}_{n}^{k}\right)-\bar{u}_{n t t}^{k}=f_{n}^{k}(r, t),
$$

где $f_{n}^{k}(r, t)$ определяются из предыдущих уравнений этой системы, при этом $f_{0}^{1}(r, t) \equiv 0$.

Далее, из краевого условия (2) в силу (6), с учетом леммы 1 будем иметь

$$
\bar{u}_{n}^{k}(r, 0)=\bar{\tau}_{n}^{k}(r), \bar{u}_{n t}^{k}(r, 0)=\bar{\nu}_{n}^{k}(r), \bar{u}_{n}^{k}(1, t)=\psi_{n}^{k}(t), k=\overline{1, k_{n}}, n \geq 0 .
$$


В (11), (12) произведя замену $\bar{v}_{n}^{k}(r, t)=\bar{u}_{n}^{k}(r, t)-\psi_{n}^{k}(t)$, получим

$$
\begin{gathered}
t^{p}\left(\bar{v}_{n r r}^{k}+\frac{m-1}{r} \bar{v}_{n r}^{k}-\frac{\lambda_{n}}{r^{2}} \bar{v}_{n}^{k}\right)-\bar{v}_{n t t}^{k}=\bar{f}_{n}^{k}(r, t), \\
\bar{v}_{n}^{k}(r, 0)=\tau_{n}^{k}(r), \quad \bar{v}_{n t}^{k}(r, 0)=\nu_{n}^{k}(r), \quad \bar{v}_{n}^{k}(1, t)=0, k=\overline{1, k_{n}}, \quad n \geq 0, \\
\bar{f}_{n}^{k}(r, t)=f_{n}^{k}(r, t)+\frac{\lambda_{n}}{r^{2}} t^{p} \psi_{n}^{k}+\psi_{n t t}^{k}, \\
\tau_{n}^{k}(r)=\bar{\tau}_{n}^{k}(r)-\psi_{n}^{k}(0), \nu_{n}^{k}(r)=\bar{\nu}_{n}^{k}(r)-\psi_{n t}^{k}(0) .
\end{gathered}
$$

Произведя замену $\bar{v}_{n}^{k}(r, t)=r^{\frac{(1-m)}{2}} v_{n}^{k}(r, t)$ задачу $(13),(14)$ приведем к следующей задаче

$$
\begin{gathered}
L v_{n}^{k} \equiv t^{p}\left(v_{n r r}^{k}+\frac{\bar{\lambda}_{n}}{r^{2}} v_{n}^{k}\right)-v_{n t t}^{k}=\widetilde{f}_{n}^{k}(r, t), \\
v_{n}^{k}(r, 0)=\widetilde{\tau}_{n}^{k}(r), \quad v_{n t}^{k}(r, 0)=\widetilde{\nu}_{n}^{k}(r), v_{n}^{k}(1, t)=0,
\end{gathered}
$$

где

$$
\begin{gathered}
\bar{\lambda}_{n}=\frac{\left[(m-1)(3-m)-4 \lambda_{n}\right]}{4}, \widetilde{f}_{n}^{k}(r, t)=r^{\frac{(m-1)}{2}} \bar{f}_{n}^{k}(r, t), \\
\widetilde{\tau}_{n}^{k}(r)=r^{\frac{(m-1)}{2}} \tau_{n}^{k}(r), \widetilde{\nu}_{n}^{k}(r)=r^{\frac{(m-1)}{2}} \nu_{n}^{k}(r) .
\end{gathered}
$$

Решение задачи (15), (16) ищем в виде

$$
v_{n}^{k}(r, t)=v_{1 n}^{k}(r, t)+v_{2 n}^{k}(r, t),
$$

где $v_{1 n}^{k}(r, t)$ - решение задачи

$$
\begin{gathered}
L v_{1 n}^{k}=\widetilde{f}_{n}^{k}(r, t), \\
v_{1 n}^{k}(r, 0)=v_{1 n t}^{k}(r, 0)=0, v_{1 n}^{k}(1, t)=0,
\end{gathered}
$$

a $v_{2 n}^{k}(r, t)$ - решение задачи

$$
\begin{gathered}
L v_{2 n}^{k}=0, \\
v_{2 n}^{k}(r, 0)=\widetilde{\tau}_{n}^{k}(r), v_{2 n t}^{k}(r, 0)=\widetilde{\nu}_{n}^{k}(r), v_{2 n}^{k}(1, t)=0 .
\end{gathered}
$$

Решение выше указанных задач, рассмотрим в виде

$$
v_{n}^{k}(r, t)=\sum_{s=1}^{\infty} R_{s}(r) T_{s}(t)
$$

при этом пусть

$$
\widetilde{f}_{n}^{k}(r, t)=\sum_{s=1}^{\infty} a_{n s}^{k}(t) R_{s}(r), \tilde{\tau}_{n}^{k}(r)=\sum_{s=1}^{\infty} b_{n s}^{k} R_{s}(r), \tilde{\nu}_{n}^{k}(r)=\sum_{s=1}^{\infty} e_{n s}^{k} R_{s}(r) .
$$

Подставляя (22) в (18), (19), с учетом (23), получим задачу

$$
\begin{gathered}
R_{s r r}+\frac{\overline{\lambda_{n}}}{r^{2}} R_{s}+\mu R_{s}=0,0<r<1, \\
R_{s}(1)=0,\left|R_{s}(0)\right|<\infty, \\
T_{s t t}+\mu t^{p} T_{s}(t)=-a_{n s}(t), 0<t<\alpha, \\
T_{s}(0)=0, T_{s t}(0)=0 .
\end{gathered}
$$


Ограниченным решением задачи $(24),(25)$ является [11]

$$
R_{s}(r)=\sqrt{r} J_{\nu}\left(\mu_{s, n} r\right),
$$

где $\nu=\frac{n+(m-2)}{2}, \mu=\mu_{s, n}^{2}$.

Задача $(26),(27)$ сводится к интегральному уравнению Вольтерра второго рода относительно $T_{s, n}(t)[1]$

$$
T_{s, n}(t)+\mu_{s, n}^{2} \int_{0}^{t}(t-\xi) \xi^{p} T_{s, n}(\xi) d \xi=-\int_{0}^{t}(t-\xi) a_{n s}(\xi) d \xi
$$

которое имеет, и притом единственное, решение.

Подставляя (28) в (23) получим

$$
\begin{gathered}
r^{-\frac{1}{2}} \widetilde{f}_{n}^{k}(r, t)=\sum_{s=1}^{\infty} a_{n s}^{k}(t) J_{\nu}\left(\mu_{s} r\right), r^{-\frac{1}{2}} \widetilde{\tau}_{n}^{k}(r)=\sum_{s=1}^{\infty} b_{n s}^{k} J_{\nu}\left(\mu_{s} r\right), \\
r^{-\frac{1}{2}} \widetilde{\nu}_{n}^{k}(r)=\sum_{s=1}^{\infty} e_{n s}^{k} J_{\nu}\left(\mu_{s} r\right), 0<r<1 .
\end{gathered}
$$

Ряды (30) - разложение в ряды Фурье-Бесселя [12], если

$$
\begin{aligned}
a_{n s}^{k}(t) & =2\left[J_{\nu+1}\left(\mu_{s, n}\right)\right]^{-2} \int_{0}^{1} \sqrt{\xi} \widetilde{f}_{n}^{k}(\xi, t) J_{\nu}\left(\mu_{s, n} \xi\right) d \xi, \\
b_{n s}^{k} & =2\left[J_{\nu+1}\left(\mu_{s, n}\right)\right]^{-2} \int_{0}^{1} \sqrt{\xi} \widetilde{\tau}_{n}^{k}(\xi) J_{\nu}\left(\mu_{s, n} \xi\right) d \xi, \\
e_{n s}^{k} & =2\left[J_{\nu+1}\left(\mu_{s, n}\right)\right]^{-2} \int_{0}^{1} \sqrt{\xi} \widetilde{\nu}_{n}^{k}(\xi) J_{\nu}\left(\mu_{s, n} \xi\right) d \xi,
\end{aligned}
$$

где $\mu_{s, n}, s=1,2, \ldots$ - положительные нули функций Бесселя $J_{\nu}(z)$, расположенные в порядка возрастания их величины.

Из (28), (29) получим решение задачи (18), (19) в виде

$$
v_{1 n}^{k}(r, t)=\sum_{s=1}^{\infty} \sqrt{r} T_{s, n}(t) J_{\nu}\left(\mu_{s, n} r\right)
$$

где $a_{n s}^{k}(t)$ определяется из (31).

Далее, подставляя (28) в (20), (21), с учетом (23), получим задачу

$$
\begin{gathered}
V_{s t t}+\mu_{s, n}^{2} t^{p} V_{s}(t)=0,0<t<\alpha, \\
V_{s}(0)=b_{n s}^{k}, V_{s t}(0)=e_{n s}^{k},
\end{gathered}
$$

в которой, произведя замену

$$
T_{s}(t)=V_{s}(t)-b_{n s}^{k}-t e_{n s}^{k}
$$

приходим к следующей задаче

$$
\begin{gathered}
T_{s t t}+\mu_{s, n}^{2} t^{p} T_{s}(t)=-q_{n s}^{k}(t), \\
T_{s}(0)=0, T_{s t}(0)=0, \\
q_{n s}^{k}(t)=\mu_{s, n}^{2} t^{p}\left(b_{n s}^{k}+t e_{n s}^{k}\right) .
\end{gathered}
$$


Задачи (35), (36) сводится также к интегральному уравнению (29), где вместо $a_{n s}^{k}(t)$ берется $q_{n s}^{k}(t)$.

Из (28), (29), (34) найдем решение задачи (20), (21)

$$
v_{2 n}^{k}(r, t)=\sum_{s=1}^{\infty} \sqrt{r} V_{s, n}(t) J_{\nu}\left(\mu_{s, n} r\right)
$$

где $b_{n s}^{k}, e_{n s}^{k}$ находятся из (32).

Следовательно, сначала решив задачу (8), $(12)(n=0)$, а затем (9), $(12)(n=1)$ и т.д., найдем последовательно все $v_{n}^{k}(r, t)$ из $(17)$, где $v_{1 n}^{k}(r, t)$, $v_{2 n}^{k}(r, t)$ определяются из (33), (37), $k=\overline{1, k_{n}}, n \geq 0$.

Итак, в области $D_{\alpha}$, имеет место

$$
\int_{H} \rho(\theta) L u d H=0
$$

Пусть $f(r, \theta, t)=R(r) \rho(\theta) T(t)$, причем $R(r) \in V_{0}, V_{0}$ - плотна в $L_{2}((0,1))$, $\rho(\theta) \in C^{\infty}(H)$ - плотна в $L_{2}(H)$, а $T(t) \in V_{1}, V_{1}$ - плотна в $L_{2}((0, \alpha))$. Tогда $f(r, \theta, t) \in V, V=V_{0} \otimes H \otimes V_{1}$ - плотна в $L_{2}\left(D_{\alpha}\right)$ [13].

Отсюда и из (38), следует, что

$$
\int_{D_{\alpha}} f(r, \theta, t) L u d D_{\alpha}=0
$$

и

$$
L u=0, \forall(r, \theta, t) \in D_{\alpha} .
$$

Таким образом, решением задачи (1) является ряд

$$
u(r, \theta, t)=\sum_{n=0}^{\infty} \sum_{k=1}^{k_{n}}\left\{\psi_{n}^{k}(t)+r^{\frac{(1-m)}{2}}\left[v_{1 n}^{k}(r, t)+v_{2 n}^{k}(r, t)\right]\right\} Y_{n, m}^{k}(\theta),
$$

где $v_{1 n}^{k}(r, t), v_{2 n}^{k}(r, t)$ находятся из $(33),(37)$.

Имеют место следующие свойства нулей функций Бесселя [12]:

1. Если $\mu_{\nu, 1}, \mu_{\nu, 2}, \ldots-$ положительные нули функций $J_{\nu}(z)$, упорядоченные по возрастанию значений, то

$$
0<\mu_{\nu, 1}<\mu_{\nu+1,1}<\mu_{\nu, 2}<\mu_{\nu+1,2}<\mu_{\nu, 3}<\ldots, \nu>-1 .
$$

2. Пусть $\mu_{\nu}, \mu_{\nu}^{\prime}, \mu_{\nu}^{\prime \prime}$ являются наименьшими положительными нулями функций $J_{\nu}(z), J_{\nu}^{\prime}(z), J_{\nu}^{\prime \prime}(z)$ соответственно. Тогда

$$
\begin{gathered}
\sqrt{\nu(\nu+2)}<\mu_{\nu}<\sqrt{2(\nu+1)(\nu+3)}, \nu>0, \\
\sqrt{\nu(\nu+2)}<\mu_{\nu}^{\prime}<\sqrt{2 \nu(\nu+1)}, \nu>0, \\
\sqrt{\nu(\nu-1)}<\mu_{\nu}^{\prime \prime}<\sqrt{\left(\nu^{2}-1\right)}, \nu>1 .
\end{gathered}
$$

Также справедливы формулы $[2,12]$ :

$$
\begin{gathered}
\sin z=z\left(1-z \sum_{n=1}^{\infty}\left(4 n^{2}-1\right)^{-1}\left[J_{n}(n z)\right]^{2}\right), \\
J_{\nu}(z)=\sqrt{\frac{2}{\pi z}} \cos \left(z-\frac{\pi}{2} \nu-\frac{\pi}{4}\right)+0\left(\frac{1}{z^{3 / 2}}\right), \nu \geq 0, \\
2 J_{\nu}^{\prime}(z)=J_{\nu-1}(z)-J_{\nu+1}(z) .
\end{gathered}
$$


Применяя признак Даламбера и упомянутые свойства, доказывается, что ряды (33), (37) и продифференцированные ряды сходятся абсолютно и равномерно. Далее используя формулы (40) и оценки [7]

$$
\left|k_{n}\right| \leq c_{1} n^{m-2},\left|\frac{\partial^{q}}{\partial \theta_{j}^{q}} Y_{n, m}^{k}(\theta)\right| \leq c_{2} n^{\frac{m}{2}-1+q}, j=\overline{1, m-1}, q=0,1, \ldots,
$$

а также леммы и ограничения на коэффициенты уравнения (1) и на заданные функции $\tau(r, \theta), \nu(r, \theta), \psi(t, \theta)$, показывается, что полученное решение (39) принадлежит классу $C^{1}\left(\bar{D}_{\alpha}\right) \cap C^{2}\left(D_{\alpha}\right)$.

Следовательно, разрешимость задачи 1 установлена.

\section{3. ЕДИНСТВЕННОСТЬ РЕШЕНИЯ ЗАДАЧИ 1}

Для этого сначала построим решение краевой задачи для сопряженного уравнения $\left(1^{*}\right)$ с данными

$$
\left.v\right|_{\Gamma_{\alpha}}=0,\left.v\right|_{S_{\alpha}}=0,\left.v_{t}\right|_{S_{\alpha}}=\nu(r, \theta)=\bar{\nu}_{n}^{k}(r) Y_{n, m}^{k}(\theta), k=\overline{1, k_{n}}, n \geq 0,
$$

где $\bar{\nu}_{n}^{k}(r) \in G, G$ - множество функций $\nu(r)$ из класса $C([0,1]) \cap C^{1}((0,1))$. Множество $G$ плотно всюду в $L_{2}((0,1))$ [13].

Решение задачи $\left(1^{*}\right),(42)$ будем искать в виде $(6)$, где функции $\bar{v}_{n}^{k}(r, t)$ будут определены ниже. Тогда, аналогично п. 2 , функции $\bar{v}_{n}^{k}(r, t)$ удовлетворяют систему уравнений (8)-(10), где $\tilde{a}_{i n}^{k}, a_{i n}^{k}, \tilde{b}_{n}^{k}$ заменены соответственно на $-\tilde{a}_{i n}^{k},-a_{i n}^{k},-\tilde{b}_{n}^{k}$, а $\tilde{c}_{n}^{k}$ на $\tilde{d}_{n}^{k}, i=1, \ldots, m, k=\overline{1, k_{n}}, n \geq 0$.

Далее, из краевого условия (42), в силу (6), получим

$$
\bar{v}_{n}^{k}(1, t)=0, \bar{v}_{n}^{k}(r, \alpha)=0, \bar{v}_{n t}^{k}(r, \alpha)=\bar{\nu}_{n}^{k}(r), k=\overline{1, k_{n}}, \quad n=0,1, \ldots .
$$

Как ранее замечено, каждое уравнение системы (8)-(10) представимо в виде (11). Как в п. 2, нетрудно показать, что задача (11), (43) имеет также единственное решение.

Таким образом, решение задачи $\left(1^{*}\right),(42)$ в виде ряда (39) построено, которое в силу $(40),(41)$ принадлежит классу $C^{1}\left(\bar{D}_{\alpha}\right) \cap C^{2}\left(D_{\alpha}\right)$.

Из определения сопряженных операторов $L, L^{*}[14]$ имеем

$$
v L u-u L^{*} v=-v P(u)+u P(v)-u v Q,
$$

где

$$
\begin{gathered}
P(u)=t^{p} \sum_{i=1}^{m} u_{x_{i}} \cos \left(N^{\perp}, x_{i}\right)-u_{t} \cos \left(N^{\perp}, t\right), \\
Q=\sum_{i=1}^{m} a_{i} \cos \left(N^{\perp}, x_{i}\right)-b \cos \left(N^{\perp}, t\right),
\end{gathered}
$$

а $N^{\perp}$ - внутреняя нормаль к границе $\partial D_{\alpha}$. По формуле Грина, имеем

$$
\int_{D_{\alpha}}\left(v L u-u L^{*} v\right) d D_{\alpha}=\int_{\partial D_{\alpha}}\left[\left(v \frac{\partial u}{\partial N}-u \frac{\partial v}{\partial N}\right) M+u v Q\right] d s
$$


где

$$
\begin{aligned}
\frac{\partial}{\partial N} & =t^{p} \sum_{i=1}^{m} \cos \left(N^{\perp}, x_{i}\right)-\cos \left(N^{\perp}, t\right) \frac{\partial}{\partial t}, \\
M^{2} & =t^{2 p} \sum_{i=1}^{m} \cos ^{2}\left(N^{\perp}, x_{i}\right)+\cos ^{2}\left(N^{\perp}, t\right) .
\end{aligned}
$$

Из (44), принимая во внимание однородные граничные условия (2) и условия (42) получим

$$
\int_{S_{\alpha}} \nu(r, \theta) u(r, \theta, \alpha) d s=0 .
$$

Поскольку линейная оболочка система функций $\left\{\bar{\nu}_{n}^{k}(r) Y_{n, m}^{k}(\theta)\right\}$ плотна $L_{2}\left(S_{\alpha}\right)$ [13], то из (45) заключаем, что $u(r, \theta, \alpha)=0, \forall(r, \theta) \in S_{\alpha}$.

Таким образом, мы приходим к задаче Дирихле:

$$
L u=0,\left.u\right|_{S_{0}}=0,\left.u\right|_{\Gamma_{\alpha}}=0,\left.u\right|_{S_{\alpha}}=0,
$$

которая имеет нулевое решение, если выполняется условие (4) [3].

Следовательно, единственность решения задачи 1 установлена и теорема 1 доказана.

Замечание 1. Отметим, что корректность смешанной задачи для многомерных гиперболических уравнений показана в [15].

\section{ЛИТЕРАТУРА}

1. Бицадзе А. В. Некоторые классы уравнений в частных производных. Москва: Наука, 1981. 448 с.

2. Тихонов А. Н., Самарский А. А. Уравнения математической физики. Москва: Наука, 1966. 724 с.

3. Алдашев С. А. Корректность задач Дирихле и Пуанкаре в цилиндрической области для вырождающихся многомерных гиперболических уравнений с оператором Геллерстедта. Нелинейные колебания. 2014. № 4. С. 3-12.

4. Алдашев С. А. Корректность задач Дирихле и Пуанкаре в цилиндрической области для вырождающихся многомерных гиперболических уравнений с оператором Чаплыгина. Научные ведомости БелГУ. Математика. Физика. 2012. Вып. 26. № 5 (124). С. 12-25.

5. Краснов М. Л. Смешанные краевые задачи для вырождающихся линейных гиперболических дифференциальных уравнений второго порядка. Матем. сб. 1959. T. 49 (91). C. $29-84$.

6. Барановский Ф. Т. Смешанная задача для линейного гиперболического уравнения второго порядка, вырождющегося на начальной плоскости. Ученые записки Ленингр. пед. института. 1958. Т. 183. С. 23-58.

7. Михлин С.Г. Многомерные сингулярные интегралы и интегральные уравнения. Москва: Физматгиз, 1962. 254 с.

8. Алдашев С. А. О задачах Дарбу для одного класса многомерных гиперболических уравнений. Дифферени. уравнения. 1998. Т. 34. № 1. С. 64-68. 
9. Алдашев С. А. Краевые задачи для многомерных гиперболических и смешанных уравнений. Алматы: Гылым, 1994. 170 с.

10. Алдашев С. А. Критерий существования собственных функций спектральной задачи Дарбу-Проттера для вырождающихся многомерных гиперболических уравнений. Дифферени. уравнения. 2005. Т. 41. № 6. С. 795-801.

11. Камке Э. Справочник по обыкновенным дифференциальным уравнениям. Москва: Наука, 1965. 703 с.

12. Бейтмен Г., Эрдейи А. Высшие трансцендентные функции. Т. 2. Москва: Наука, 1974. 295 с.

13. Колмогоров А. Н., Фомин С. В. Элементы теории функций и функционального анализа. Москва: Наука, 1976. 543 с.

14. Смирнов В. И. Курс высшей математики. Т. 4. Ч. 2. Москва: Наука, 1981. 550 c.

15. Алдашев С. А. Корректность смешанной задачи для многомерных гиперболических уравнений с волновым оператором. Укр. матем. журнал. 2017. Т. 69. № 7. С. 992-999.

Поступила: 01.02.2019 / Принята: 24.05.2019 